\title{
A flexible modelling framework leading to a probabilistic multiaxial Kitagawa-Takahashi diagram: Applied to cast Al-Si alloys
} \author{
Etienne Pessard ${ }^{1}$ and Pierre Osmond ${ }^{2}$ \\ ${ }^{1}$ LAMPA, Arts et métiers ParisTech, 49000 Angers, France \\ ${ }^{2}$ PSA Peugeot Citröen, 92256 La Garenne-Colombes, France \\ ${ }^{3}$ I2M, Arts et métiers ParisTech, 33405 Talence Cedex, France
}

Viet-duc Le ${ }^{1,2,3}$, Daniel Bellett ${ }^{1, a}$, Franck Morel ${ }^{1}$, Nicolas Saintier $^{3}$, Thierry Palin-Luc ${ }^{3}$,

\begin{abstract}
The aim of this work is to propose simple analytical tools to predict the fatigue strength of cast aluminium components as a function of the casting process and post-cast treatment. The proposed methodology is based on the Murakami approach to predict the maximum defect size and a flexible modelling framework which leads to the construction of a probabilistic, multiaxial Kitagawa-Takahashi diagram. This framework is capable of modelling two independent co-existing fatigue damage mechanisms. This methodology will be applied to fatigue data taken from the literature as well as tests conducted on AlSi7 cast specimens manufactured via three different processes.
\end{abstract}

\section{Introduction}

For cast aluminium alloys, it has been demonstrated that microstructural characteristics and heterogeneities are the principal factors affecting the fatigue strength. Figure 1 shows a typical microstructural image of a cast hypo-eutectoid Al-Si alloy, in which these can be seen (i.e. casting defects, micro-shrinkage or gas porosity, the eutectic zones containing Si-particles, intermetallic inclusions and the aluminium matrix characterised by the DAS and SDAS). These microstructural heterogeneities strongly depend on the manufacturing process (i.e. gravity die casting, lost foam casting) and the post-casting treatment (T6, T7 and/or HIP - Hot Isostatic Pressing).

The aim of this paper is (a) to propose a flexible modelling framework to predict the fatigue strength as a function of defect size and (b) to apply this to cast Al-Si alloys, in combination with the methodology proposed by Murakami [1] to characterize the casting defect size. The ultimate aim of this work is to propose simple analytical tools to predict the fatigue strength of components manufactured by different casting processes and post-cast treatments.

\footnotetext{
a Corresponding author: daniel.bellett@ensam.eu
}

This is an Open Access article distributed under the terms of the Creative Commons Attribution License 4.0, which permits unrestricted use, distribution, and reproduction in any medium, provided the original work is properly cited. 


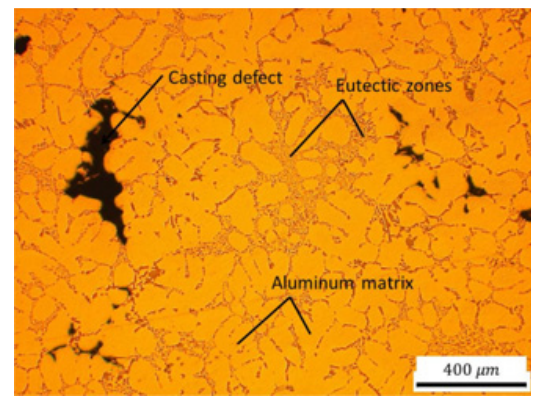

Figure 1. Typical microstructure of a cast Al-Si alloy.

\section{The Murakami methodology for defect size characterisation}

The defect size of metallic materials is often characterised by microscopic observations of polished samples. However, it has been showed that the maximum observed defect size, using this technique, is generally smaller than the defect size that leads to the fatigue failure (or the defect size observed on the fatigue failure surfaces). In response to this finding, Murakami [1] proposed a method to characterise the defect size distribution, using simple optical microscopic observations, and to then predict the maximum defect size in a given surface area or volume. This approach is based on Extreme Value Theory in which the Gumbel distribution is used to characterise the distribution of maximum defect size. In this article, this approach has been applied to cast aluminium alloys fabricated by different casting process and heat treatments, so as to obtain significantly differed degrees of porosity. It is shown that, in general, the method is capable of predicting a maximum defect size, which is of the same order of magnitude as the defect size that causes final fatigue.

\section{Probabilistic fatigue strength modelling}

Koutiri [2] highlighted the co-existence of two distinct high cycle fatigue damage mechanisms in gravity die cast AlSi7Cu05Mg03-T7 alloys. The first mechanism is one of crack initiation due to local plasticity in the alpha dendrites or the eutectic zones and the second is due to crack propagation from shrinkagepores. The dominant mechanism depends on the defect size (and consequently on the casting or postcasting process). A probabilistic modelling framework has been developed by the authors which is capable of reflecting this competition. In this framework the damage mechanisms are modelled using two different fatigue criteria. By using a Weibull distribution to define the threshold quantity of each criterion, it can be shown that the probability of failure for the two mechanisms is given by:

$$
P_{F 1}=1-\exp \left[-\left(\frac{\sigma_{e q}}{\sigma_{e q}^{\prime}}\right)^{m_{1}}\right] \quad \text { and } \quad P_{F 2}=1-\exp \left[-\left(\frac{\Delta K}{\Delta K_{\mathrm{th} 02}^{\prime}}\right)^{m_{2}}\right] \text {. }
$$

The competition between the two mechanisms is then combined via the weakest link concept:

$$
1-P_{F}=\left(1-P_{F 1}\right)\left(1-P_{F 2}\right) \text {. }
$$

This framework leads to a Kitagawa-Takahashi type diagram that is highly flexible, probabilistic and can take into account multiaxial loading conditions. 


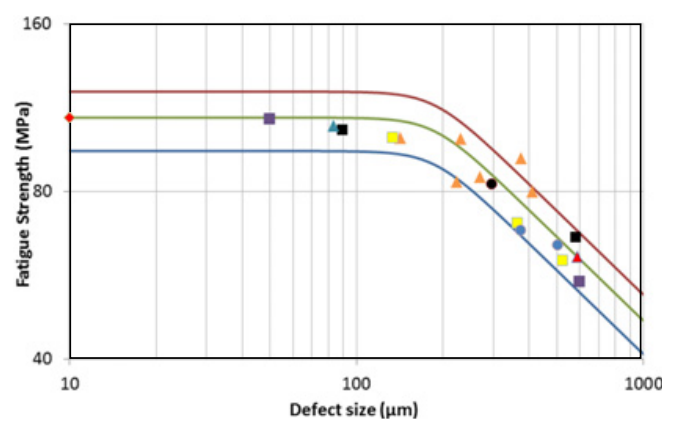

Figure 2. Comparison between the predicted fatigue strength and experimental data take from the literature for cast Al-Si alloys subjected to uniaxial $\mathrm{R}=-1$ loads.

\section{Results}

Figure 2 shows the application of this framework to fatigue data taken from the literature (uniaxial $\mathrm{R}=-1$ loads) for cast AlSi7 materials manufactured by different processes. The red, green and blue lines are the predications for $90 \%, 50 \%$ and $10 \%$ probability of failure. It can be seen that the predictions are in good agreement with the experimental data.

\section{References}

[1] Y. Murakami, Effects of Small Defects and Nonmetallic Inclusions (2002) 321-332

[2] I. Koutiri, D. Bellett, F. Morel, L. Augustin, J. Adrien, Int. J. Fatigue 47 (2013) 44-57

[3] E. Pessard, D. Bellett, F. Morel, I. Koutiri, Eng. Fract. Mech 109 (2013) 89-104 